\title{
Detection of a persistent-current qubit by resonant activation
}

\author{
P. Bertet, ${ }^{1}$ I. Chiorescu, ${ }^{1, *}$ K. Semba,,${ }^{1,2}$ C. J. P. M. Harmans, ${ }^{1}$ and J. E. Mooij ${ }^{1}$ \\ ${ }^{1}$ Quantum Transport Group, Kavli Institute of Nanoscience, Delft University of Technology, Lorentzweg 1,2628CJ, \\ Delft, The Netherlands \\ ${ }^{2}$ NTT Basic Research Laboratories, NTT Corporation, Atsugi-shi, Kanagawa 243-0198, Japan
}

(Received 29 April 2004; published 20 September 2004)

\begin{abstract}
We present the implementation of a scheme to detect the quantum state of a persistent-current qubit. It relies on the dependency of the measuring superconducting quantum interference device plasma frequency on the qubit state, which we detect by resonant activation. With a measurement pulse of only 5 ns, we observed Rabi oscillations with high visibility $(65 \%)$.
\end{abstract}

DOI: 10.1103/PhysRevB.70.100501

PACS number(s): 74.50.+r, 03.67.Lx, 85.25.Cp

Superconducting circuits containing Josephson junctions are promising candidates to be the building blocks (qubits) for future quantum computers. ${ }^{1}$ Coherent dynamics have been observed with several different qubit designs. Complex sequences of rotations on the Bloch sphere could be performed in NMR-like experiments: Rabi, Ramsey, spin-echo, and composite pulse sequences were demonstrated. ${ }^{2-5}$ In these experiments, the detection of the qubit energy state is done after the coherent operations have been accomplished by reading out a macroscopic detector with an output that is correlated to the qubit energy eigenstate. By averaging the signal delivered, one infers the qubit eigenstate occupation probability.

A hysteretic dc superconducting quantum interference device (SQUID) has been succesfully employed to measure the state of a flux qubit ${ }^{5}$ (a similar scheme was used in Ref. 3 in the case of a split Cooper pair box). In this experiment, a fast dc current pulse (DCP) sent through the SQUID induced switching to the finite-voltage state conditional on the qubit state. In this Rapid Communication, we propose and demonstrate a readout method, faster than the previous one, which does not require a large bandwidth line to inject current through the SQUID and thus will allow a stronger filtering of the bias lines. The method is based on the dependence of the SQUID plasma frequency on the qubit's state measured by a resonant activation microwave pulse (RAP). We compare Rabi oscillations obtained by the two schemes in exactly the same conditions, and show that the RAP method significantly increases the contrast.

The persistent-current qubit consists of a micron-size superconducting loop intersected with three Josephson junctions. ${ }^{6}$ It is threaded by a magnetic flux $\Phi_{x}$ generated by an external coil. When the total phase across the three junctions $\gamma_{q}$ is close to $\pi$ (meaning that $\Phi_{x}$ is close to $\Phi_{0} / 2$ ), the loop has two low-energy eigenstates (ground state $|0\rangle$ and excited state $|1\rangle$ ) well separated from the higher-energy ones, and can thus be used as a qubit. They are linear combinations of two states $|\uparrow\rangle$ and $|\downarrow\rangle$ that carry an average persistentcurrent of $+I_{p}$ and $-I_{p}$ and are coupled by tunneling. The effective Hamiltonian reads $H=-h\left(\epsilon \sigma_{z} / 2-\Delta \sigma_{x} / 2\right)$ in the $\{|\uparrow\rangle,|\downarrow\rangle\}$ basis, where $h \Delta / 2$ is the tunneling matrix element between the two basis states, and $\epsilon=2 I_{p}\left(\Phi_{x}-\Phi_{0} / 2\right) / h$. This leads to an energy separation between the two states $E_{1}$ $-E_{0} \equiv h f_{q}=h \sqrt{\Delta^{2}+\epsilon^{2}}$.
The average current in state $|i\rangle \quad(i=0,1) \quad I_{i}$ $=-\left\langle\partial H / \partial \Phi_{x}\right\rangle_{i}$ can be computed from the previous equations. It is shown in the inset of Fig. 1(c) for the parameters of our sample. The qubit is inductively coupled to a hysteretic dc SQUID whose critical current depends on $I_{i}$ by an amount $M I_{i} \alpha$, where $M$ is the mutual inductance between the qubit and the SQUID (including both kinetic and geometric contributions), and $\alpha=\partial I_{C} / \partial \Phi_{x}$ is the slope of the SQUID modulation curve. The requirement imposed upon any detection scheme is to detect the small (about 2\%) variation in the SQUID critical current associated with a transition between the qubit states in a time shorter than the qubit's energy relaxation time $T_{1}$. A method was demonstrated in Refs. 3 and 5. It consists of applying a short DCP to the SQUID at a value $I_{b}$ during a time $\Delta t$, so that the SQUID will switch out of its zero-voltage state ${ }^{8,9}$ with a probability $P_{s w}\left(I_{b}\right)$. For well-chosen parameters, the detection efficiency $D$ $=\max I_{b}\left|P_{s w}^{1}-P_{s w}^{0}\right|$, where $P_{s w}^{0}\left(P_{s w}^{1}\right)$ is the switching probability if the qubit is in the ground (excited) state, can approach 1. The switching probability then directly measures the qubit's energy level population.

The $\mathrm{Al} / \mathrm{AlO}_{x} / \mathrm{Al}$ tunnel junctions as well as the SQUID and qubit's aluminum loops were obtained by e-beam lithography and shadow evaporation on an oxidized silicon substrate. The SQUID was shunted by an on-chip aluminum capacitor and connected to the current injection line through a $400 \Omega$ on-chip gold resistor [see Fig. 1(a)]. Both current and voltage lines were filtered by copper-powder filters, ${ }^{10}$ yielding a rise time of $10 \mathrm{~ns}$ for the bias current pulses. The voltage across the SQUID was amplified at room temperature and sampled by an acquisition card. By repeating the measurements typically 10000 times at a rate of $25 \mathrm{kHz}$ (allowing the system, including the qubit, to relax to its ground state between successive experimental sequences), we derive the switching probability. Pulsed microwave irradiation was applied to the qubit through a coaxial cable attenuated by $20 \mathrm{~dB}$ at $T=1.5 \mathrm{~K}$. The rise time of the microwave pulses was $500 \mathrm{ps}$. The sample was enclosed in a copper box thermally anchored to the mixing chamber of our dilution refrigerator (base temperature $25 \mathrm{mK}$ ) to shield it from RF noise.

The parameters of our qubit were determined by fitting spectroscopic measurements with the above formulas. For 


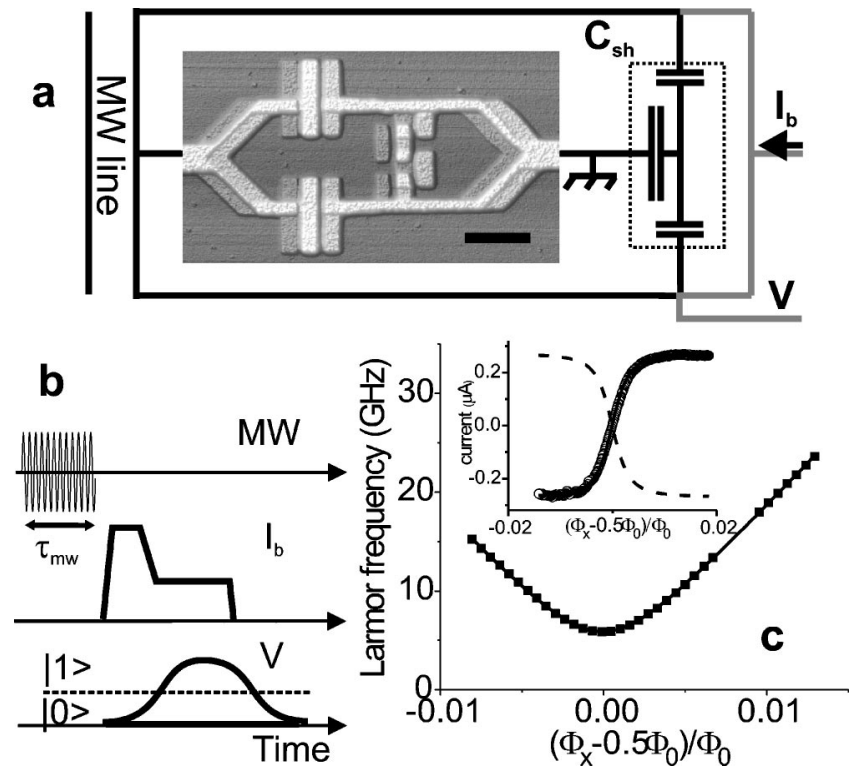

FIG. 1. (a) Atomic-force microscope (AFM) picture of the sample (the scale bar indicates $1 \mu \mathrm{m}$ ). Two layers of aluminum were evaporated under $\pm 20^{\circ}$ with an oxidation step in between. The Josephson junctions are formed at the overlap areas between the two images. The qubit is the small loop containing three Josephson junctions; the SQUID is the outer loop and contains two large junctions. The SQUID is shunted by a capacitor $C_{s h}=12 \mathrm{pF}$ connected by aluminum leads of inductance $L=170 \mathrm{pH}$ (solid black line). The current is injected through a resistor (gray line) of $400 \Omega$. Microwave pulses are applied via a shortcut ("MW line" in the figure). (b) DCP measurement method: the microwave pulse induces the desired Bloch sphere rotations. It is followed by a current pulse of duration $20 \mathrm{~ns}$, whose amplitude $I_{b}$ is optimized for the best detection efficiency. A 400 ns lower-current plateau follows the DCP and keeps the SQUID in the running state to facilitate the voltage pulse detection. (c) Larmor frequency of the qubit and (inset) persistent current in the ground state versus external flux. The squares and (inset) the circles are experimental data. The solid lines are numerical adjustments giving the tunneling matrix element $\Delta$, the persistent current $I_{p}$, and the mutual inductance $M$. The dashed line in the inset is the calculated persistent current in the excited state.

$\Delta=5.855 \mathrm{GHz}, I_{p}=272 \mathrm{nA}$, the agreement is excellent [see Fig. 1(c)]. We also determined the coupling constant between the SQUID and the qubit by fitting the qubit "step" appearing in the SQUID's modulation curve ${ }^{7}$ [see inset of Fig. 1(c)] and found $M=20 \mathrm{pH}$. We first performed Rabi oscillation experiments with the DCP detection method [Fig. 1(b)]. We chose a bias point $\Phi_{x}$, tuned the microwave frequency to the qubit resonance, and measured the switching probability as a function of the microwave pulse duration $\tau_{m w}$. The observed oscillatory behavior [Fig. 2(a)] is a proof of the coherent dynamics of the qubit.

The contrast of the Rabi oscillations was observed to strongly depend on the exact bias point $\Phi_{x}$, ranging from 5 to $40 \%$. This visibility was lower than in our previous experiment. ${ }^{5}$ We optimized the amplitude $I_{b}$ of the current pulse for each $\Phi_{x}$ by measuring the switching probability as a function of $I_{b}$ both without microwave $\left[P_{s w}^{0}\left(I_{b}\right)\right]$ and after a $\pi$ pulse $\left[P_{s w}^{\pi}\left(I_{b}\right)\right]$. Typical data are shown in Fig. 2(b). The solid black curve $P_{t h}^{0}\left(I_{b}\right)$ is a fit to $P_{s w}^{0}\left(I_{b}\right)$ assuming that switching occurs by thermal activation across the potential barrier $^{9}$ with an escape temperature of $200 \mathrm{mK}$. The dashed line $P_{t h}^{1}\left(I_{b}\right)$ was calculated with the same parameters assuming that the qubit is always in state $|1\rangle$. The curve $P_{s w}^{\pi}\left(I_{b}\right)$ is then well approximated by a weighted sum of the curves $P_{t h}^{1}(I)$ and $P_{t h}^{0}\left(I_{b}\right)$ with coefficients $p_{0}=0.68$ and $p_{1}=0.32$ (gray solid line). The maximum difference between the two curves $P_{t h}^{1}(I)$ and $P_{t h}^{0}\left(I_{b}\right)$ being $85 \%$, we conclude that the detection efficiency $D$ of our detector should be of this order, but that the excited state of our qubit is only populated up to $32 \%$ when switching to the finite-voltage state occurs. Thus, it seems that the qubit partially relaxes towards its ground state during the measurement process. As we will show below, this problem does not occur with the alternative measurement scheme described hereafter.

The SQUID, connected to the capacitor $C_{s h}$ by aluminum wires of inductance $L$, behaves as an oscillator with a characteristic frequency called the plasma frequency, $2 \pi f_{p}=[(L$ $\left.\left.+L_{J}\right) C_{s h}\right]^{-1 / 2}$. This frequency depends on the bias current $I_{b}$ and on the critical current $I_{C}$ via the Josephson inductance $L_{J}=\Phi_{0} /\left(2 \pi I_{C} \sqrt{1-I_{b}^{2} / I_{C}^{2}}\right)$. Thus, the plasma frequency takes different values $f_{p}^{(0)}$ or $f_{p}^{(1)}$ depending on the qubit's state. This effect has already been observed in Ref. 11 by measuring the transmission of a weak probe at a frequency close to $f_{p}$ while keeping the SQUID in the zero-voltage state. With a probe of larger power, it has recently been demonstrated that a Josephson junction could switch between two distinct oscillation states while staying in the zero-voltage regime, due to its nonlinearity. ${ }^{12}$ Such a bifurcation amplifier could be used to detect the state of a charge qubit. ${ }^{13}$ Here we apply a microwave pulse at a frequency close to $f_{p}^{0}$. We adjust the power so that the SQUID switches to the finite voltage state by resonant activation ${ }^{14}$ if the qubit is in state $|0\rangle$, whereas it stays in the zero-voltage state if it is in state $|1\rangle$. If the two resonant activation peaks corresponding to the frequencies $f_{p}^{0}$ and $f_{p}^{1}$ are distinct enough, the switching probability of the SQUID measures the probability that the qubit is in the state $|0\rangle$. Similarly, in Ref. 4 , the quantum state of a current-biased Josephson junction (phase qubit) has been detected by applying a microwave pulse that induces switching determined by the qubit state. In our experiment, the detector is distinct from the qubit. Although switching to the finite-voltage state is a dissipative process potentially harmful for quantum computation, the specific interest of this scheme is that it allows to heavily filter the bias-current line since no fast dc or rf pulse is needed there.

A typical resonant activation peak is shown in the inset of Fig. 3. Its width depends on the frequency, ranging between 20 and $50 \mathrm{MHz}$. This corresponds to a quality factor between 50 and 150 . The peak has an asymmetric shape, with a very sharp slope on its low-frequency side and a smooth highfrequency tail, due to the SQUID nonlinearity. We were able to qualitatively recover these features by simple numerical simulations using the RCSJ model. ${ }^{15}$ The resonant activation peak can be unambiguously distinguished from environmental resonances by its dependence on the magnetic flux threading the SQUID loop $\Phi_{s q}$. Figure 3 shows the measured peak frequency for different fluxes around $\Phi_{s q}=1.5 \Phi_{0}$, together 

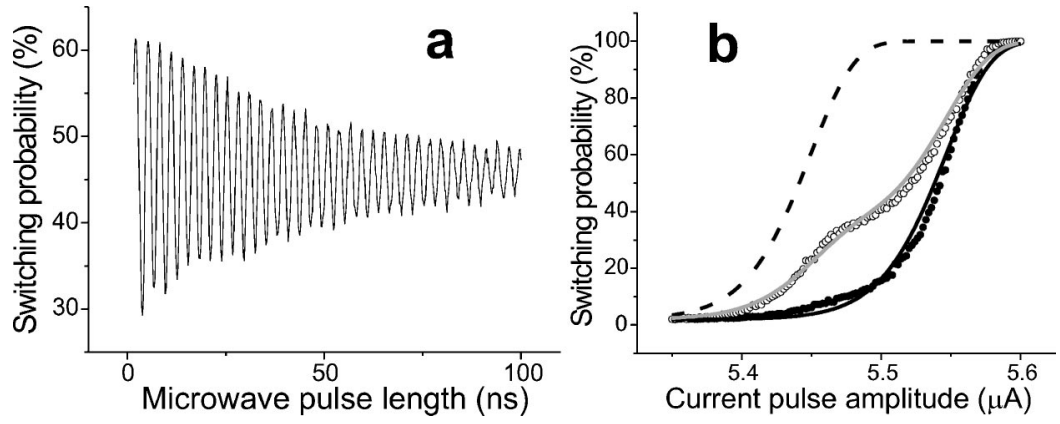

FIG. 2. (a) Rabi oscillations at a Larmor frequency $f_{q}=7.15 \mathrm{GHz}$ measured with the DCP method. (b) Switching probability as a function of current pulse amplitude $I_{b}$ without [closed circles, curve $P_{s w}^{0}\left(I_{b}\right)$ ] and with [open circles, curve $P_{s w}^{\pi}\left(I_{b}\right)$ ] a $\pi$ pulse applied. The solid black line $P_{t h}^{0}\left(I_{b}\right)$ is a numerical adjustment to $P_{s w}^{0}\left(I_{b}\right)$ assuming escape in the thermal regime (escape temperature $T=200 \mathrm{mK}$ ). The dashed line [curve $P_{t h}^{1}\left(I_{b}\right)$ ] is calculated with the same parameters for a critical current $100 \mathrm{nA}$ smaller, which would be the case if state $|1\rangle$ was detected with probability unity. The gray solid line is the sum $0.32 P_{t h}^{1}\left(I_{b}\right)+0.68 P_{t h}^{0}\left(I_{b}\right)$.

with the measured switching current (dashed line). The solid line is a numerical fit to the data using the formula $f_{p}$ $=\left(2 \pi \sqrt{\left\{L+\Phi_{0} /\left[2 \pi I_{C}\left(\Phi_{s q}\right)\right]\right\} C_{s h}}\right)^{-1}$. From this fit we deduce the following values $C_{s h}=12 \pm 2 \mathrm{pF}$ and $L=170 \pm 20 \mathrm{pH}$, close to the design. We are thus confident that the observed resonance is due to the plasma frequency.

We then measure the effect of the qubit on the resonant activation peak. The principle of the experiment is sketched in Fig. 4(a). A first microwave pulse at the Larmor frequency induces a Rabi rotation by an angle $\theta_{1}$. A second microwave pulse of duration $\tau_{2}=10 \mathrm{~ns}$ is applied immediately after, at a frequency $f_{2}$ close to the plasma frequency, with a power high enough to observe resonant activation. In this experiment, we apply a constant bias current $I_{b}$ through the SQUID $\left(I_{b}=2.85 \mu \mathrm{A}, I_{b} / I_{C}=0.85\right)$ and maintain it at this value $500 \mathrm{~ns}$ after the microwave pulse to keep the SQUID in the running state for a while after switching occurs. This allows sufficient voltage to build up across the SQUID and makes detection easier, similarly to the plateau used at the end of the DCP in the previously shown method. At the end of the experimental sequence, the bias current is reduced to zero in

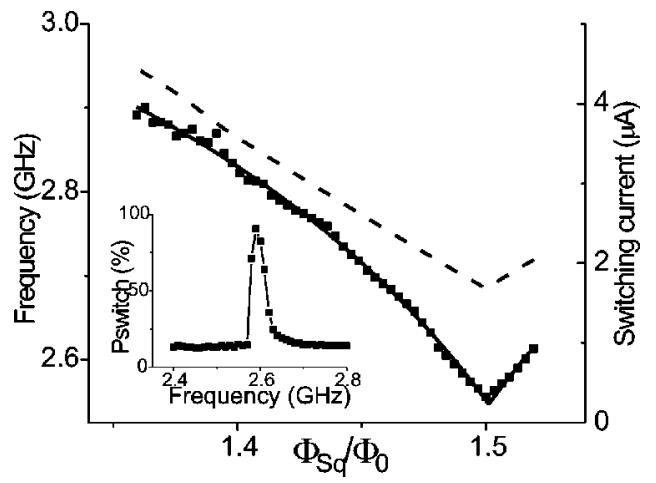

FIG. 3. (Inset) Typical resonant activation peak (width $40 \mathrm{MHz}$ ), measured after a $50 \mathrm{~ns}$ microwave pulse. Due to the SQUID nonlinearity, it is much sharper at low than at high frequencies. (Figure) Center frequency of the resonant activation peak as a function of the external magnetic flux (squares). It follows the switching current modulation (dashed line). The solid line is a fit yielding the values of the shunt capacitor and stray inductance given in the text. order to retrap the SQUID in the zero-voltage state. We measured the switching probability as a function of $f_{2}$ for different Rabi angles $\theta_{1}$. The results are shown in Fig. 4(b). After the microwave pulse, the qubit is in a superposition of the states 0 and 1 with weights $p_{0}=\cos ^{2}\left(\theta_{1} / 2\right)$ and $p_{1}$ $=\sin ^{2}\left(\theta_{1} / 2\right)$. Correspondingly, the resonant activation signal
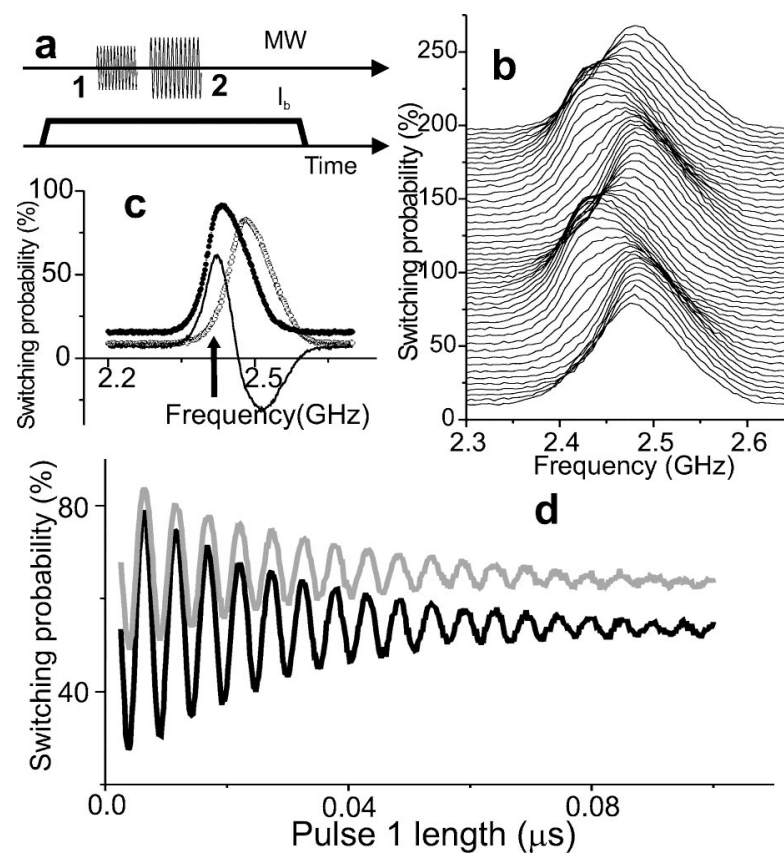

FIG. 4. (a) Principle of the RAP detection scheme. After the Rabi pulse, a microwave pulse at the plasma frequency resonantly enhances the escape rate. The bias current is maintained for $500 \mathrm{~ns}$ above the retrapping value. (b) Resonant activation signal for different Rabi angles. Each curve was offset by $5 \%$ for visibility. The Larmor frequency was $f_{q}=8.5 \mathrm{GHz}$. Pulse 2 duration was $10 \mathrm{~ns}$. (c) Resonant activation peak without (full circles) and after (open circles) a $\pi$ pulse. The continuous line is the difference between the two switching probabilities. The black arrow indicates the frequency at which the data of Fig. 4(d) were taken. (d) Rabi oscillation measured by dc current pulse (gray line, amplitude $A=40 \%$ ) and by resonant activation method with a 5 ns RAP (black line, $A$ $=62 \%$ ), at the same Larmor frequency. 
is a sum of two peaks centered at $f_{p}^{0}$ and $f_{p}^{1}$ with weights $p_{0}$ and $p_{1}$, which reveal the Rabi oscillations.

We show the two peaks corresponding to $\theta_{1}=0$ (curve $P_{s w}^{0}$, full circles) and $\theta_{1}=\pi$ (curve $P_{s w}^{\pi}$, open circles) in Fig. 4(c). They are separated by $f_{p}^{0}-f_{p}^{1}=50 \mathrm{MHz}$ and have a similar width of $90 \mathrm{MHz}$. This is an indication that the $\pi$ pulse efficiently populates the excited state (any significant probability for the qubit to be in $|0\rangle$ would result into broadening of the curve $\left.P_{s w}^{\pi}\right)$. The difference between the two curves $S(f)=P_{s w}^{0}-P_{s w}^{\pi}$ [solid line in Fig. 4(c)] gives a lower bound of the excited state population after a $\pi$ pulse. Because of the above-mentioned asymmetric shape of the resonant activation peaks, it yields larger absolute values on the low- than on the high-frequency side of the peak. Thus the plasma oscillator nonlinearity increases the sensitivity of our measurement, which is reminiscent of the ideas exposed in Ref. 13. On the data shown here, $S(f)$ attains a maximum $S_{\max }$ $=60 \%$ for a frequency $f_{2}^{*}$ indicated by an arrow in Fig. 4(c). The value of $S_{\max }$ strongly depends on the microwave pulse duration and power. The optimal settings are the result of a compromise between two constraints: a long microwave pulse provides a better resonant activation peak separation, but, on the other hand, the pulse should be much shorter than the qubit damping time $T_{1}$, to prevent loss of excited state population. Under optimized conditions, we were able to reach $S_{\max }=68 \%$.

Finally, we fixed the frequency $f_{2}$ at the value $f_{2}^{*}$ and measured Rabi oscillations [black curve in Fig. 4(d)]. We compared this curve to the one obtained with the DCP method in exactly the same conditions (gray curve). The contrast is significantly improved, while the dephasing time is evidently the same. This enhancement is partly explained by the rapid 5 ns RAP [for the data shown in Fig. 4(d)] compared to the $30 \mathrm{~ns}$ DCP. But we cannot exclude that the DCP intrinsically increases the relaxation rate during its rise time. Such a process would be in agreement with the fact that for these bias conditions, $T_{1} \simeq 100 \mathrm{~ns}$, three times longer than the DCP duration.

In conclusion, we demonstrated a method for detecting the state of a persistent-current qubit. We exploited the dependence of the SQUID's plasma frequency on the qubit's state, which we measured by applying a microwave pulse that induces resonant activation. It provided large detection efficiency even for short microwave pulses (5 ns). The contrast of the Rabi oscillations was mainly limited by the resonant activation peaks separation. By improving the quality factor of the plasma oscillator and using composite pulses, we might thus reach the single-shot regime while keeping the bias current line heavily filtered and having a large dephasing time.

We thank Y. Nakamura for direct input in this work, F. Balestro, and A. Lupascu for fruitful discussions, and R. Schouten and B. ven den Enden for technical support. P.B. acknowledges support from the European Community.
* Present address: Department of Physics and Astronomy, Michigan State University, East Lansing, MI 48824, USA.

${ }^{1}$ Y. Makhlin, G. Schoen, and A. Shnirman, Rev. Mod. Phys. 73, 357 (2001).

${ }^{2}$ Y. Nakamura, Yu. A. Pashkin, and J. S. Tsai, Nature (London) 398, 786 (1999).

${ }^{3}$ D. Vion, A. Aassime, A. Cottet, P. Joyez, H. Pothier, C. Urbina, D. Esteve, and M. H. Devoret, Science 296, 886 (2002).

${ }^{4}$ J. M. Martinis, S. Nam, J. Aumentado, and C. Urbina, Phys. Rev. Lett. 89, 117901 (2002).

${ }^{5}$ I. Chiorescu, Y. Nakamura, C. J. P. M. Harmans, and J. E. Mooij, Science 299, 1869 (2003).

${ }^{6}$ J. E. Mooij, T. P. Orlando, L. Levitov, L. Tian, C. H. van der Wal, and S. Lloyd, Science 285, 1036 (1999).

${ }^{7}$ C. H. van der Wal, A. C. J. ter Haar, F. K. Wilhelm, R. N. Schouten, C. J. P. M. Harmans, T. P. Orlando, Seth Lloyd, and J. E. Mooij, Science 290, 773 (2000).

${ }^{8}$ A. Cottet, D. Vion, A. Aassime, P. Joyez, D. Esteve, and M. H.
Devoret, Physica C 367, 197 (2002).

${ }^{9}$ F. Balestro, J. Claudon, J. P. Pekola, and O. Buisson, Phys. Rev. Lett. 91, 158301 (2003).

${ }^{10}$ J. M. Martinis, M. H. Devoret, and J. Clarke, Phys. Rev. B 35, 4682 (1987).

${ }^{11}$ A. Lupascu, C. J. M. Verwijs, R. N. Schouten, C. J. P. M. Harmans, and J. E. Mooij, cond-mat/0311510 (unpublished).

${ }^{12}$ I. Siddiqi, R. Vijay, F. Pierre, C. M. Wilson, L. Frunzio, M. Metcalfe, C. Rigetti, R. J. Schoelkopf, M. H. Devoret, D. Vion, and D. Esteve, cond-mat/0312553 (unpublished).

${ }^{13}$ I. Siddiqi, R. Vijay, F. Pierre, C. M. Wilson, M. Metcalfe, C. Rigetti, L. Frunzio, and M. H. Devoret, cond-mat/0312623 (unpublished).

${ }^{14}$ M. H. Devoret, J. M. Martinis, D. Esteve, and J. Clarke, Phys. Rev. Lett. 53, 1260 (1984).

${ }^{15}$ M. H. Devoret, D. Esteve, J. M. Martinis, A. Cleland, and J. Clarke, Phys. Rev. B 36, 58 (1987). 